\title{
Software educacional: simulador de ventilação mecânica e seus efeitos hemodinâmicos
}

\author{
Educational software: mechanical ventilation simulator and its hemodynamic effects
}

Moyses de Campos Junior ${ }^{1}$ (D) $\mid$ mcjunior@pucsp.br

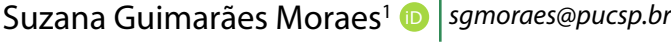

\section{RESUMO}

Introdução: O manejo adequado da ventilação mecânica (VM) é considerado de alta complexidade, e o uso de um simulador permite a aquisição de habilidades em ambiente seguro.

Objetivo: O presente trabalho desenvolveu um software que simula o manejo da VM e sua interação cardiopulmonar.

Método: Na elaboração do software, utilizou-se a Web Hipertext Markup Language (HTML) versão 5. Um engenheiro de software avaliou a qualidade técnica do simulador, e o conteúdo foi avaliado por especialistas da área, com o uso de instrumento de percepção com escala de Likert de concordância de cinco pontos.

Resultado: O simulador desenvolvido disponibilizou referências teóricas, escolha de situações clínicas, alteração de parâmetros de ventilação, monitorização de mecânica ventilatória e hemodinâmica, gasometria arterial e animações. A qualidade foi aprovada pelo engenheiro de software. $\mathrm{Na}$ avaliação do conteúdo por especialistas, a média geral dos pontos de Likert (L) e concordância (Co) para as assertivas foram: para "disponível para aluno on-line" ( $L=4,29$ e $C o=85,7 \%)$, para "disponível para aluno extraclasse" $(L=4,856$ e $C o=100 \%)$, para "estimular o raciocínio" $(L=5$ e Co $=100 \%)$, para "excesso de conteúdo" $(L=3,14$ e $C o=28,6 \%)$ e para "fácil de usar" $(L=3,43$ e Co $=71,4 \%)$. O índice de validade do conteúdo médio foi igual a 0,86 . Corrigiram-se os erros encontrados pelos especialistas. A concordância para disponibilidade do simulador para aluno exigiu melhora da ajuda nos parâmetros de ventilação, nas condutas não VM, dois níveis de dificuldade e diminuição da poluição visual.

Conclusão: Os especialistas consideraram esse simulador como uma ferramenta facilitadora para o ensino da interação cardiopulmonar na VM.

Palavras-chave: Software; Simulador Interativo; Ventilação Mecânica; Monitorização Hemodinâmica.

\section{ABSTRACT}

Introduction: The appropriate management of mechanical ventilation (MV) is considered of high complexity, and the use of a simulator allows students to acquire these abilities in a safe environment.

Objective: The study developed an educational software that simulates MV management and its cardiopulmonary interaction.

Method: The software was developed with Web Hypertext Markup Language version 5 (HTML5). Software engineers evaluated the technical quality and experts assessed the content using a perception tool with a five-point Likert scale of agreement.

Result: The developed simulator offered theoretical references, a choice of clinical condition, adjustable ventilation settings, monitoring of ventilatory and hemodynamic mechanicals, arterial gasometry, and animations. Its quality was approved by a software engineer. In the expert content evaluation, the following Likert $(L)$ and agreement $(A)$ average scores for the statements were found: for "available online for students" ( $L=4.29$ and $A=85.7 \%)$, for "available to students outside the class" ( $L=4.856$ and $A=100 \%)$, for "stimulates reasoning" ( $L=5$ and $A=100 \%)$, for "excess content" ( $L=3.14$ and $A=28.6 \%)$ and for "user friendly" ( $L=3.43$ and $A=71.4 \%)$. The average content validity index was 0.86 . The errors found by the experts were corrected. Agreement in relation to availability of the simulator for students required improved help in the ventilation settings, in nonMV actions, two levels of difficulty, and less visual pollution.

Conclusion: Experts considered this simulator a facilitating tool for teaching cardiopulmonary interaction on mechanical ventilation. Keywords: Software; Simulation Training; Mechanical Ventilation; Hemodynamic Monitoring.

${ }^{1}$ Pontifícia Universidade Católica de São Paulo, Sorocaba, São Paulo, Brasil.

Editora-chefe: Rosiane Viana Zuza Diniz.

Editor associado: Antonio da Silva Menezes Junior.

Recebido em 08/09/21; Aceito em 24/10/21.

Avaliado pelo processo de double blind review. 


\section{INTRODUÇÃO}

A aprendizagem na área de ventilação mecânica (VM) é considerada de alta complexidade, e o ambiente de simulação se mostrou útil na introdução dos princípios básicos da mecânica pulmonar e na interação entre o paciente e o ventilador ${ }^{1-4}$. A simulação com o uso de um sistema microprocessado composto de manequim, gases, ventilador mecânico e monitores torna o ambiente mais próximo da realidade, porém, apesar de mais convidativo, exige investimento financeiro para manutenção adequada de todos os seus componentes, bem como regras para acesso controlado e supervisionado ${ }^{4}$. O software simulador computacional de VM abriu uma possibilidade de acesso irrestrito e livre na internet. O simulador Xlung possibilita alterar os parâmetros e permite a correlação entre as situações clínicas, as variáveis de VM e a alteração na oximetria de pulso e na gasometria arterial em tempo real ${ }^{3}$. O ensino de VM para alunos sem a visualização da hemodinâmica e/ou de parâmetros estáticos pode ser utilizado dependendo dos objetivos específicos de ensino da graduação.

A pandemia da coronavirus disease 2019 (Covid-19) aumentou as internações por síndrome respiratória aguda e exigiu recursos e capacitação de profissionais para uso da VM. O ambiente de simulação, no estágio mais avançado do ensino, deve chamar a atenção para as possíveis intercorrências (hipotensão, atividade elétrica sem pulso, aspiração, vazamento e pneumotórax), bem como para a estratégia mais adequada para enfrentamento dessas situações ${ }^{5-7}$. O cenário de uma simulação possibilita ao aluno testar seus conhecimentos em ambiente seguro, sem riscos para os pacientes ${ }^{8}$. Se mais dados visuais estão disponíveis, o educador deixa de ser o senhor do conteúdo para ser um dos participantes na condução dessa linha terapêutica de suporte ${ }^{9}$.

O objetivo deste trabalho foi o desenvolvimento de um simulador com interação cardiopulmonar que disponibilizasse um centro cardiorrespiratório, capnografia, hemodinâmica, animações de ventilação (alvéolo-capilar e planos torácicos), intercorrências e opções terapêuticas não ventilatórias.

\section{MÉTODOS}

\section{Elaboração de textos}

Elaboraram-se o Termo de Consentimento Livre e Esclarecido (TCLE), textos de ajuda para o entendimento do manejo da VM e ilustrações que explicavam as definições mais importantes na literatura existente, com o objetivo de facilitar o aprendizado autodirigido ${ }^{5,10-20}$. Os voluntários avaliaram o software com o instrumento de percepção, por meio de uma escala de concordância de Likert de cinco pontos, respondendo a todas as asserções ${ }^{21}$. Nesse questionário, os usuários puderam relatar aspectos positivos e negativos, comentários, críticas e sugestões que julgaram importantes ${ }^{22,23}$.

\section{Desenvolvimento do software}

Após a autorização pelo Comitê de Ética e Pesquisa (CEP) da Faculdade de Ciências Médicas e da Saúde de Sorocaba Parecer no 3.434.803 e Certificado de Apresentação para Apreciação Ética (CAAE) no 16188219.9.0000.5373, de 3 de julho de 2019 -, o software foi desenvolvido com apoio de um funcionário da Divisão de Tecnologia da Informação desta universidade, utilizando a Web Hipertext Markup Language versão 5 (HTML5) com o editor livre Notepad++ versão 7.6.6., Javascript para elaboração de cálculos, Cascading Style Sheets (CSS) para os estilos e Canvas do HTML para as animações e os gráficos ${ }^{24,25}$. O software foi publicado num site da instituição.

\section{Sujeitos da pesquisa}

Após autorização do CEP, o software foi disponibilizado aos voluntários (engenheiro e especialistas da área da saúde) via e-mail: para o simulador (link, usuário e senha), manual e link para TCLE e instrumento de percepção. Na avaliação do simulador, adotaram-se os seguintes procedimentos:

- Um engenheiro de software examinou a qualidade do simulador.

- A análise do conteúdo foi feita por especialistas fisioterapeutas ou médicos intensivistas que tivessem trabalhado com VM nos últimos dois anos e/ou com experiência docente na área.

\section{Avaliação da qualidade do software pela tecnologia da informação}

A avaliação técnica do simulador foi realizada por engenheiro de acordo com a Norma Brasileira (NBR) 13596, que determinou as características de qualidade do software. Essa norma foi traduzida pela Associação Brasileira de Normas Técnicas (ABNT) a partir da Norma 9126 da International Organization for Standardization em conjunto com a International Electrotechnical Commission (ISO/IEC), que é constantemente revisada pela $\mathrm{ISO}^{26,27}$. A qualidade em uso pode ser medida por meio da operação do produto em condição de uso normal ou simulada, verificando-se a existência e o nível das características e subcaracterísticas definidas na Norma ISO/ IEC 9126 (NBR13596) $^{27}$.

\section{Análise dos dados do instrumento de percepção}

Foi associada uma escala atitudinal: concordo plenamente $(C P)$, concordo $(C)$, indiferente (I), discordo (D) e discordo plenamente (DP). Asserções com impacto positivo 
tiveram valores diferentes na escala de Likert, quando comparadas às asserções negativas:

- Positivas: $C P=5, C=4, I=3, D=2$ e $D P=1$.

- Negativas: $C P=1, C=2, I=3, D=4$ e $D P=5$.

Os cálculos para o instrumento de percepção do software, visando possibilitar a aplicação de estatística paramétrica, foram:

- Média dos pontos em escala de Likert para cada asserção.

- Concordância é a soma das opiniões com pontos atribuídos iguais a quatro ou cinco, divididos pelo total das opiniões para cada assertiva e depois convertidos em porcentagem ${ }^{28}$.

- $\quad$ índice de validade do conteúdo (IVC) foi obtido pela soma das opiniões com pontos atribuídos iguais a quatro ou cinco, dividido pelo total de opiniões para cada assertiva ${ }^{28}$. O IVC geral foi obtido pela média todas assertivas.

\section{RESULTADOS}

\section{Simulador desenvolvido}

Os parâmetros básicos para simulação (formulário azul) permitem a escolha pelo usuário dentro de certos limites ou opções: grau de dificuldade, diagnóstico principal, segundo diagnóstico, sexo, idade, altura, posição no leito (importante nas simulações de SARA), temperatura, dosagem de hemoglobina e pressão barométrica. A mudança do nível de dificuldade para "HARD" randomiza a presença do índice de massa corpórea (IMC) maior que 30, pneumotórax, vazamento e aspiração, ausentes no nível "NORMAL". Os parâmetros modificados de forma indireta, após escolha do diagnóstico principal, foram: complacência pulmonar estática, resistência de vias aéreas, relação entre pressão parcial de oxigênio e fração inspirada de oxigênio $\left(\mathrm{paO}_{2} / \mathrm{FiO}_{2}\right)$, espaço morto fisiológico ("P.Dead Space"), estado de hidratação (quando se adiciona um segundo diagnóstico), volume sistólico do ventrículo esquerdo (VS), pontos de inflexão inferior e superior, resistência vascular sistêmica (RVS), randomização de aparecimento das complicações como aspiração e/ou pneumotórax. O valor do IMC e da capacidade residual funcional foi recalculado na mudança de opção feminino/masculino ${ }^{14}$. O valor do IMC superior ou igual a 30 foi considerado elevado ${ }^{12}$. Algumas alterações param a simulação ventilatória e ativam o botão "Confirme/Start" e desabilitam o botão "Stop". O botão "Precisa Ajuda?" permite acesso aos botões de "Referências", "Ajuda" e "Alarmes e Opções", e o botão "Fechar Ajuda?" oculta o acesso nesses itens (Figura 1).

Como a simulação do centro cardiorrespiratório (CCR) periférico e central não é visível, seguiram-se os princípios de fisiologia para permitir a autorregulação nas simulações ${ }^{29}$. O CCR interfere diretamente nas frequências cardíaca e respiratória, no VS, na RVS e na resistência vascular sistêmica pulmonar (RVP). Uma falta de interação entre o CCR e a VM pode provocar assincronias.

Os parâmetros de ventilação (formulário bege) têm cinco opções de modo ventilatório ${ }^{12}$. No modo ciclado a volume (VCV), assistido controlado, há a opção de usar fluxo

Figura 1. Formulários dos parâmetros básicos para simulação (azul), parâmetros de ventilação (bege), gasometria arterial e índices (rosa), oxímetro, gráfico do monitor de ECG/PA invasiva, animação da ventilação pulmonar no plano axial (esquerda), plano sagital (direita) e alvéolo-capilar.

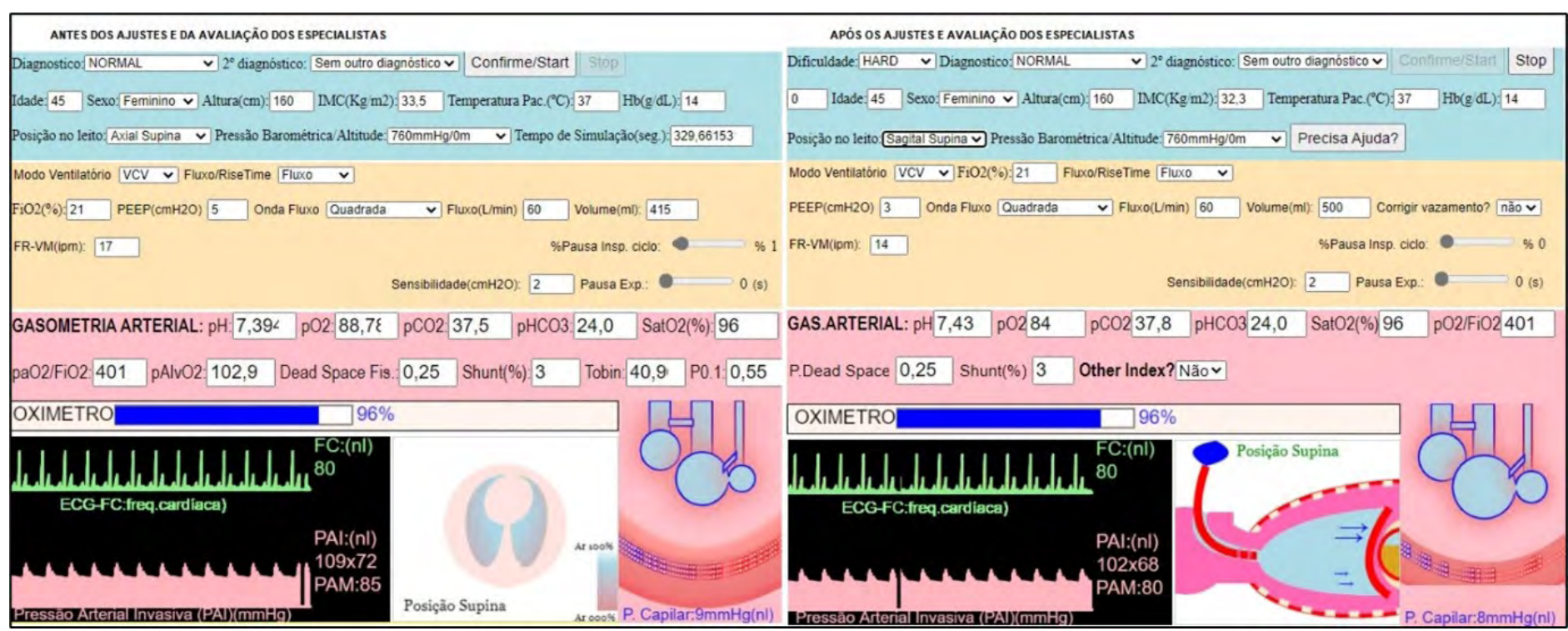

Fonte: Simulador feito pelo autor. 
ou tempo de rampa ("Fluxo/Rise Time") e o tipo de onda de fluxo. No modo ciclado a tempo com pressão controlada (PCV), assistido controlado, usa-se o rise time, com a opção de utilizar insuflação intratraqueal de gás (TGI), se necessário ${ }^{15}$. No modo pressão de suporte (PS), espontânea assistida, há a opção de ajuste do backup de apneia (modo PCV). O modo de pressão em dois níveis de pressão (BIPAP), não invasivo assistido, usa o modo PCV, o valor da pressão inspiratória é o valor máximo, e não há backup de apneia. O modo CPAP é não invasivo espontâneo e oferece a pressão positiva contínua nas vias aéreas e a $\mathrm{FiO}_{2}$. Quando se muda qualquer parâmetro, exceto a pausa inspiratória, a simulação ventilatória para e necessita confirmação no botão "Confirme/Start" (Figura 1). A opção "Corrigir vazamento?" aparece na dificuldade "HARD".

A gasometria arterial e os índices (formulário rosa) são influenciados pelo gasto energético calculado pela fórmula de Harris-Benedict, pela hemodinâmica, pela ventilação e pelas condutas. A fórmula de Weir, considerando o quociente respiratório igual a 0,8 , estimou a produção de dióxido de carbono e o consumo de oxigênio ${ }^{20}$. O modo assistido tem um acréscimo de $10 \%$ no gasto energético em relação ao controlado de ventilação ${ }^{20}$. A equação de Henderson-Hassdelbalch foi utilizada para cálculo do $\mathrm{pH}$. A saturação gasométrica arterial de oxigênio $\left(\mathrm{SaO}_{2} \%\right)$ foi obtida por meio da equação de Kelman a partir dos dados obtidos por Severinghaus, utilizando $\mathrm{paO}_{2^{\prime}}$ $\mathrm{pCO}_{2}, \mathrm{pH}$ e temperatura ${ }^{30}$. Adotou-se o método de Fick para calcular a saturação gasométrica venosa central de oxigênio $\left(\mathrm{SaO}_{2} \mathrm{~V}\right)$. A saturação de oxigênio de pulso $\left(\mathrm{SpO}_{2} \%\right)$, animada em "OXIMETRO", é o valor da $\mathrm{SaO}_{2} \%$ que sofre influência da pressão arterial média. Os índices de oxigenação como a $\mathrm{paO}_{2} / \mathrm{FiO}_{2}$, os de ventilação/perfusão como a pressão alveolar de oxigênio ( $\mathrm{pAlvO}_{2}$ ), shunt pulmonar (Shunt\%) e o "P.Dead Space" são calculados automaticamente. $O$ índice de Tobin foi calculado a partir da divisão do volume corrente (VC) em litros pela frequência respiratória, e a pressão de oclusão inspiratória a 0,1 segundo (P0.1) avaliou a resposta do CCR. Esse índice e essa pressão são contínuos e utilizados para desmame da VM. A opção de "Other index?" permite visualizar os índices $\mathrm{pAlvO}_{2^{\prime}}$ Tobin e P0.1 (Figura 1).

No gráfico que simula o monitor de eletrocardiograma (ECG), a pressão arterial (PA) invasiva (Figura 1), o valor da frequência cardíaca (FC) e da PA média e seus alarmes visuais são apresentados, em tempo real entre parênteses, como normal ("nl"), aumentado (" $\uparrow ")$ ou diminuído (" $\downarrow$ ").

A animação da ventilação pulmonar no plano axial (Figura 1) simula a bioimpedância com densidade pulmonar baixa (azul) até alta (avermelhado), posição no leito (supina/ prona), aspiração como coleção dourada posterior, pneumotórax como espaço pleural em branco e redução do volume de um dos pulmões, dreno torácico e barotrauma não controlado identificado quando o coletor tem bolhas saindo do dreno ${ }^{31,32}$.

A animação da ventilação pulmonar no plano sagital (Figura 1) simula a posição no leito, a movimentação dos músculos diafragma e intercostais, a aspiração de líquido gástrico no pulmão, o estômago e a estrutura abdominal, o pneumotórax como espaço pleural em branco e a redução do volume do pulmão, o tubo orotraqueal (COT) ou máscara facial, a TGI e o dreno torácico (sem coletor) e de barotrauma não controlado ${ }^{33}$.

A animação alvéolo-capilar foi representada no desenho de três alvéolos, três fluxos de hemácias e o valor da pressão capilar pulmonar (PCP) (Figura 1) 25,33. Nessa animação, podemse simular o aumento de "P.Dead Space", "Shunt\%", a atividade elétrica sem pulso (AESP), a diminuição dos bronquíolos distais durante a expiração quando a pressão positiva expiratória intrínseca está elevada, as bolhas de ar no enfisema intersticial (pneumotórax) e a rotura eminente no alvéolo da esquerda como uma esfera enchendo que depois se rompe.

O monitor ventilatório (Figura 2) simula e identifica o modo de ventilação, as quatro curvas (fluxo, volume, pressão e capnografia) e os alarmes visuais em amarelo, com exceção

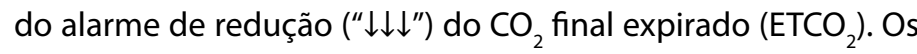
parâmetros monitorados são complacência, resistência, fluxo máximo e mínimo, pressão inspiratória máxima, pressão de platô, pressão positiva expiratória final, pressão transpulmonar (PTP), VC inspirado e expirado, VC por quilo de peso ideal, tempo do ciclo, tempo inspiratório (I) e expiratório $(E)$, relação I/E, frequência respiratória atual e demanda do CCR simulada (FRtot-pac). As curvas em tempo real de pressão X volume (PxV) ou fluxo $X$ volume (FxV) são opcionais.

A animação cardiovascular (CV) simula a contração dos ventrículos, a pulsação das artérias e veias, a AESP, os valores em tempo real da resistência vascular pulmonar (RVP) e RVS, o VS do ventrículo esquerdo, o débito cardíaco (DC), a $\mathrm{SaO}_{2} \mathrm{~V}$, a pressão venosa central (PVC), o valor randomizado do diâmetro da veia cava inferior ( $\mathrm{VCl}$ ) e sua variação (varia) para avaliação da volemia ${ }^{34}$. Os alarmes visuais para esses parâmetros seguem os mesmos padrões do ECG e a PAI (Figura 2).

Os botões de ajuda, que têm a mesma cor dos formulários, auxiliam o usuário a utilizar o simulador: nas condições gerais, parâmetros de ventilação, outras condutas e alarmes/opções. Disponibilizaram-se cinco arquivos públicos de referencial teórico, em formato em PDF, que serão abertos em outra janela, para consulta de insuficiência respiratória, diretrizes de VM, interação cardiopulmonar, transporte de $\mathrm{O}_{2} \mathrm{e}$ $\mathrm{CO}_{2}$ e VM na Covid-19 (Figura 2) ${ }^{12,35-38}$.

O formulário "Condutas Não VM" (verde, Figura 3) inclui bicarbonato, volume, sedação/analgesia, broncodilatador, 
vasopressor, além de outras condutas em certas condições: "Drenagem Torácica?" na dificuldade "HARD”, "COT Ø:" nos modos ventilatórios invasivos e "Tratar ICC?" no diagnóstico "EDEMA AGUDO".

No formulário "Alarmes e Opções" (amarelo, Figura 3), no que concerne aos valores dos alarmes, foram escolhidos os valores padrões (mínimo e máximo) para cada parâmetro, que podem ser alterados dentro de certos limites, com exceção de $\mathrm{ETCO}_{2}$ e $\mathrm{SpO}_{2}$ que apresentam apenas a opção mínima. Quando os valores determinados são ultrapassados, é ativado um som específico (ventilatório e/ou cardiovascular) e visual para alarme do parâmetro. Nas "Opções", existe a possibilidade de ativar o som, permitindo ouvir os alarmes, os batimentos cardíacos e ruídos respiratórios, a visualização da animação CV, Curva PxV, Curva FxV e o tempo de visualização dos monitores em segundos da tela (padrão de dez segundos) $)^{39}$.

\section{Avaliação do software por engenheiro de software}

Após concordância do TCLE pelo engenheiro de software, o simulador computacional foi submetido à avaliação, levando em consideração o objetivo do software e os usuários ${ }^{26,27}$. Concluiu-se que o software está apto para proposta de ensino desde que siga as necessidades mínimas para processamento dos dados, em navegador Chrome, por sua compatibilidade com Windows, Linus e Mac OS. A não observância dessa exigência poderia causar falhas. Não foi projetado para comunicação com outros softwares.

Figura 2. Monitor ventilatório, botões para ajuda e referências, curva PxV (esquerda) e animação CV (direita).
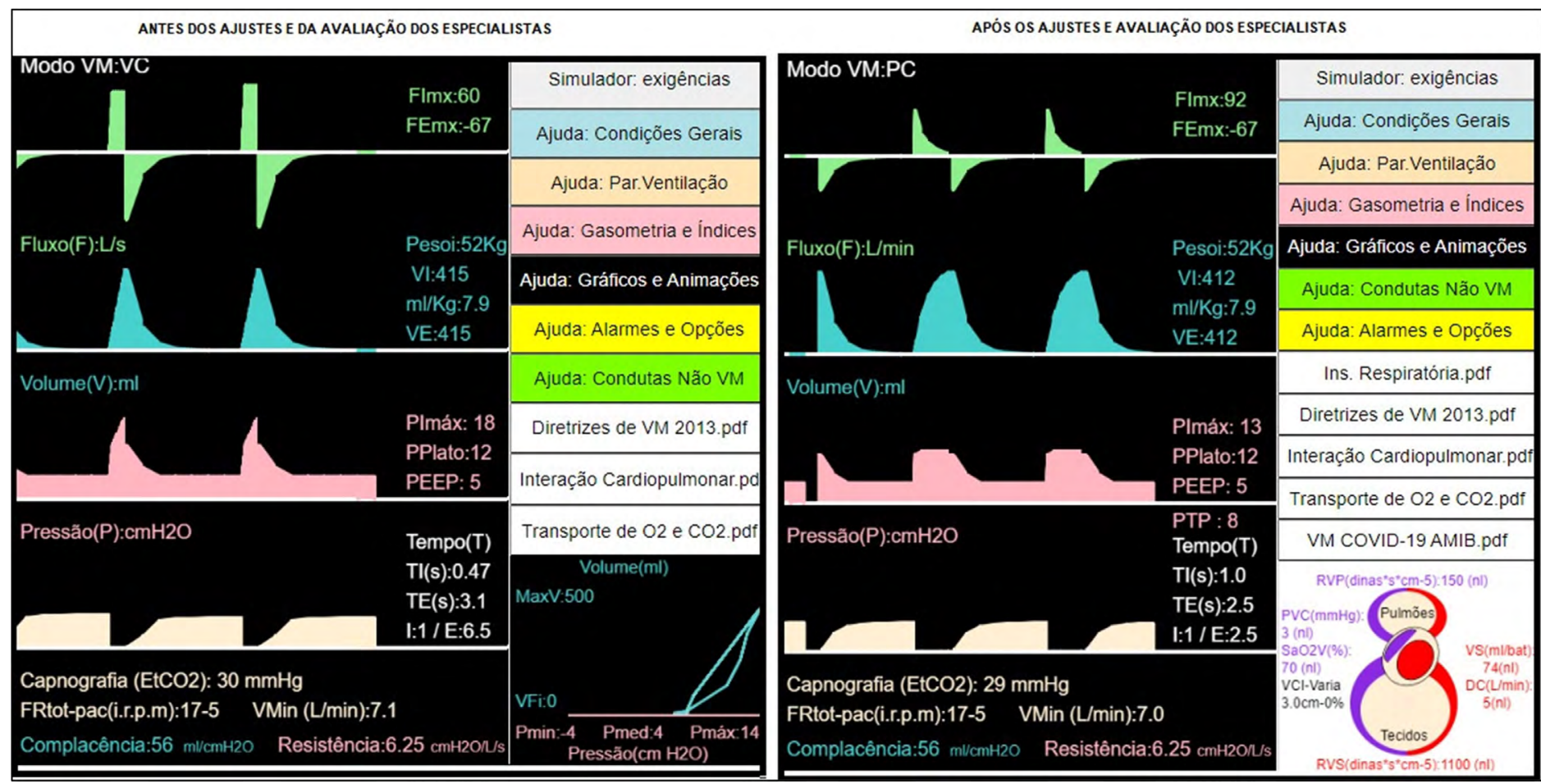

Fonte: Simulador feito pelo autor.

Figura 3. Formulários de "Condutas Não VM" e "Alarmes e Opções".

FORMULÁRIOS VERSÃO INICIAL

ALARMIES $\bigcirc$ VENTILAÇÃO MECÂNICA(VM): PInsp.max: $35 \quad$ PInsp.min: $4 \quad$ VolCor(ml Kg)max: 8 VolCor(mlKg)min: $4 \quad$ VolMin(L min)max: 10

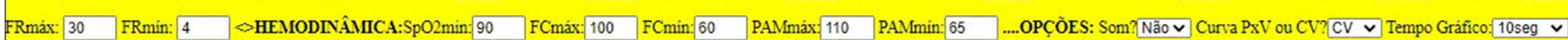
CONDUTAS Não VM: Drenagem Torácica? Não v Bicarbonato? Não v Volume EV? Não v Sedoanalgesia BNM? Sedação e Analgesia $\vee$ Broncodilatador? Não $\vee$ Vasopressor? Não $\vee$ FORMULÁRIOS VERSÄO FINAL

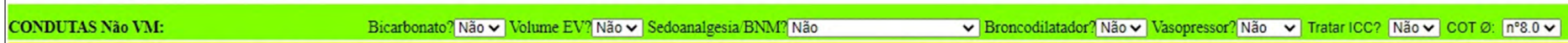
ALARIIES $\diamond$ VENTILAC̣̃̃o MIECÂNICA(VM): PInsp.máx: $35 \quad$ PInsp.min: $4 \quad \operatorname{Vol} \operatorname{Cor}(\operatorname{ml} / \mathrm{Kg}) \operatorname{máx}: 8 \quad$ VolCor(mlKg)min: $4 \quad$ VolMin(L/min)máx: 10

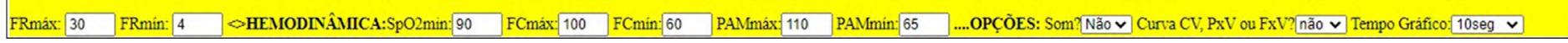
Fonte: Simulador feito pelo autor. 


\section{Avaliação do software por especialistas}

\section{Instrumento de percepção por especialistas}

Os sete especialistas (quatro fisioterapeutas e três médicos intensivistas), seis deles professores, concordaram com oTCLE, testaram o simulador e opinaram sobre o conteúdo dele (Tabela 1). Não foi imposto nenhum padrão de avaliação, permitindo aos avaliadores testar quantas vezes quisessem as mais variadas combinações de simulação no software.

Na avaliação do conteúdo por especialistas, a média dos pontos de Likert (L) e de concordância (Co) foi maior nas asserções positivas ( $L=4,45, C o=90,4 \%$ ) que nas negativas $(\mathrm{L}=4,22$, $\mathrm{Co}=77,1 \%)$. A assertiva com maior concordância foi "estimular o raciocínio" ( $L=5,00, C o=100 \%$ ), as decisivas foram "disponível on-line" ( $\mathrm{L}=4,29, \mathrm{Co}=85,7 \%)$ e "disponível extraclasse" ( $L=4,86, C o=100 \%)$, e as piores, "excesso de conteúdo" $(L=3,14, C o=28,6 \%)$ e "fácil utilização" $(L=3,43$, Co = $71,4 \%)$. Obteve-se o valor de 0,86 para a média geral do IVC.

\section{Pontos positivos, negativos e sugestões (nº de avaliadores)}

- Pontos positivos $\left(\mathrm{n}^{\circ}\right)$ : Ferramenta boa para testar teoria e/ou prática (7) e animações interessantes para interação cardiopulmonar (5) e ajuda (1).
- Pontos negativos ( $\left.n^{\circ}\right)$ : Delays nas curvas (2), visual confuso, muito poluído (4), erros de cálculos: $\mathrm{paO}_{2}$ (1) e ajuda mais detalhada para condutas não VM ou parâmetros de ventilação (4).

- Sugestões $\left(n^{\circ}\right)$ : Incluir mais referências (1), fazer testes básicos com conceitos e parâmetros novos (1), adicionar modo APRV (1) e parâmetro para identificar a hipovolemia (1).

\section{Ajustes no software simulador após opinião dos especialistas}

Após os devidos esclarecimentos sobre a interpretação dos colegas especialistas e a postura dos avaliadores para disponibilidade on-line, opções viáveis no software, os seguintes ajustes foram efetuados (Figura 4):

a) Acerto sobre atrasos (delays) na execução das curvas de monitorização.

b) Adição de mais duas referências para consulta em arquivo PDF.

c) Adição do parâmetro visual da pressão transpulmonar (PTP) quando a pausa inspiratória for maior que zero.

Tabela 1. Opinião de especialistas em relação às questões sobre o software segundo a escala de Likert. Em negrito o número total de especialistas e a opção escolhida.

\begin{tabular}{|c|c|c|c|c|c|}
\hline & DP & D & I & C & $\mathrm{CP}$ \\
\hline $\begin{array}{l}\text { 1. O conteúdo com o software pode ajudar o aluno a entender o uso racional de } \\
\text { ventilação mecânica. }\end{array}$ & & & 1 & 2 & 4 \\
\hline 2. O software deve estar à disposição dos alunos para a utilização extraclasse. & & & & 1 & 6 \\
\hline 3. A quantidade do conteúdo abordado é excessiva.an & 2 & & 2 & 3 & \\
\hline $\begin{array}{l}\text { 4. Os conteúdos abordados no software não são relevantes à formação do profissional } \\
\text { da saúde. }\end{array}$ & 5 & 1 & 1 & & \\
\hline 5. A melhor maneira de colocar o software à disposição do aluno é por acesso on-line. & & 1 & & 2 & 4 \\
\hline 6. O software é de fácil utilização. & & 2 & & 5 & \\
\hline 7. Esta metodologia de ensino permite interação entre várias disciplinas ou áreas. & & & & 3 & 4 \\
\hline 8. Esta metodologia não integra teoria e prática. ${ }^{\text {an }}$ & 5 & 2 & & & \\
\hline 9. A metodologia pode contribuir para despertar o interesse para o aprendizado. & & & & 3 & 4 \\
\hline 10. Deve ser produzido material semelhante adaptado para outras áreas ou disciplinas. & & & 1 & 1 & 5 \\
\hline 11. O material como um todo é de boa qualidade. & & & & 4 & 3 \\
\hline 12. É importante a iniciativa de avaliar uma metodologia de ensino. & & & & 1 & 6 \\
\hline 13. A metodologia de ensino estimula o raciocínio. & & & & & 7 \\
\hline 14. Esta metodologia de ensino não aproxima a área básica da área clínica. an & 5 & 2 & & & \\
\hline 15. Esta metodologia está de acordo com os objetivos da reforma curricular. & & & 2 & 3 & 2 \\
\hline 16. O software acrescenta pouco àquilo já visto em sala de aula. an & 2 & 3 & 2 & & \\
\hline $\begin{array}{l}\text { 17. A metodologia torna um assunto complexo como a ventilação mecânica mais } \\
\text { interessante. }\end{array}$ & & & 1 & 2 & 4 \\
\hline
\end{tabular}

CP: concordo plenamente, C: concordo, I: indiferente, D: discordo, DP: discordo plenamente.

Fonte: Elaborada pelos autores. 
Figura 4. Visão geral do simulador, após ajustes, com animações torácica/alvéolo-capilar e monitores, sem visualização de: alguns índices da gasometria, botões de ajuda, referências, alarmes/opções e animações (CV, PxV ou FxV).

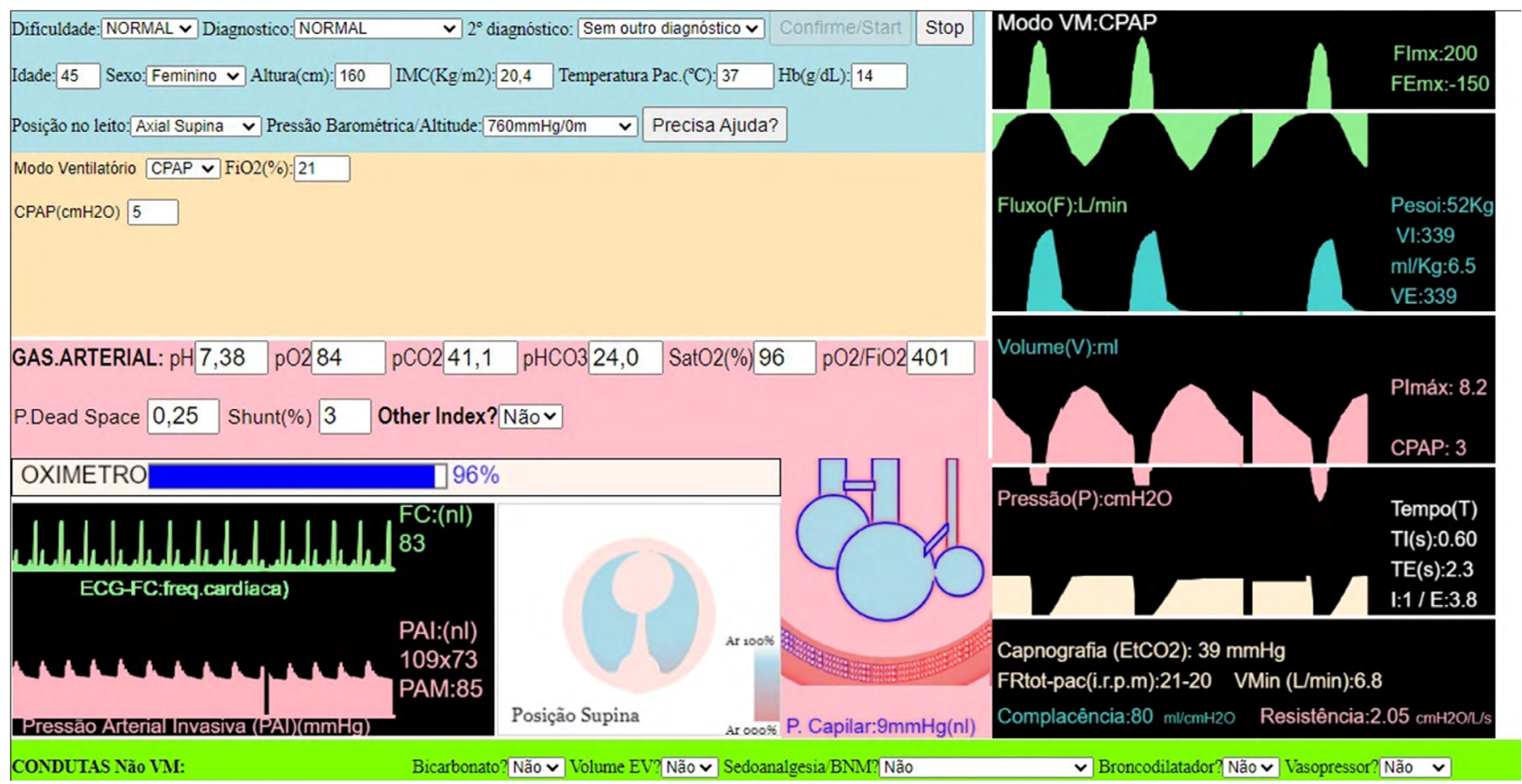

Fonte: Simulador feito pelo autor.

d) Elaborado um manual acessível no botão "Ajuda:Par. Ventilação" e mais informações no texto acessado no botão "Condutas Não VM". Dois níveis de dificuldade: "NORMAL" e"HARD".

e) Correção de cálculo da $\mathrm{paO}_{2}$.

f) Adição do diâmetro da VCl e sua variação (VCl-varia) na animação CV, para ajudar na avaliação da volemia.

g) Para diminuir a poluição visual, foram adicionados dois botões no formulário "Condições Gerais". O primeiro é "Precisa ajuda" para visualizar os botões de ajuda, alarmes/opções, referências, animação CV, curva PxV ou FxV. O segundo "Fechar ajuda" - oculta as ajudas, referências, mas não fecha as animações $\mathrm{CV}$, curvas PxV ou FxV caso tenham sido ativadas. Ocultaram-se os índices no formulário de gasometria, que podem ser visualizados se utilizar opção "Other index?".

\section{DISCUSSÃO}

A participação de médicos e fisioterapeutas contribuiu para maior êxito na condução direta da VM como descrito no consenso da Associação de Medicina Intensiva Brasileira (AMIB) de $2013^{12}$.

$\mathrm{Na}$ avaliação dos especialistas, das várias asserções, a com melhor concordância (Co) e média de Likert (L) foi "estimular o raciocínio" ( $C o=100 \%, L=5,00)^{22,23}$. As que estão na zona de alerta ( $L$ de 2,64-3,66) foram "excesso de conteúdo" ( $C o=28,6 \%, L=3,14)$ e "fácil de usar" ( $C o=71,4 \%, L=3,43)$, que são ligadas à qualidade do software ${ }^{22,23}$. A poluição visual, a velocidade de execução das curvas, o nível de dificuldade e a ajuda insuficiente foram corrigidos para disponibilidade online e/ou extraclasse. A média geral de Likert $(\mathrm{Lg})$ de todas as asserções positivas e negativas estão na zona de conforto $(\mathrm{Lg}>$ 3,66), e o índice de validação de conteúdo de 0,86 reforça que o simulador tem potencial no ensino do tema ${ }^{22,23,28}$. A usabilidade, na análise de seis simuladores, foi: 93\% para Xlung, 65\% para Hamilton, 59\% para Inter 5 Plus e Evita XL 53\%; somente Xlung tinha validação de conteúdo'.

Como não há espaço para tentativa e erro no tratamento de pacientes graves ou mesmo estáveis sob VM, a simulação de cenários clínicos é considerada uma ferramenta de aprendizagem relevante para a capacitação e aquisição de competências .

O grau de dificuldade para ensino de VM é de alta complexidade, como já havia sido relatado por Osaku². Na metodologia ativa de ensino, temos vários pontos a serem considerados, como as dificuldades e particularidades do educando, a obrigatoriedade de atingir as competências necessárias exigidas pelo plano de ensino e o tema que deve ser abordado. Na simulação, podem existir alguns problemas, e, seguindo as orientações dos saberes necessários à prática educativa de Paulo Freire ${ }^{9}$, apontamos os seguintes aspectos: 
- A dificuldade no manuseio do software pode ser atenuada pelos arquivos disponíveis para consulta, possibilidade de contato com o autor do simulador. Ensinar alunos pode exigir uma compreensão diferente das necessidades deles e respeitar o nível de entendimento. Caso as perguntas dos discentes sejam frequentes com necessidade de respostas mais detalhadas, cabe ao educador oferecer a possibilidade de encaminhar as questões por meio de chat, ao final da aula ou e-mail.

- Falta de curiosidade sobre o tema de parte dos discentes ou de todo o grupo: O educador deve esclarecer com detalhes as simulações e entender que o silêncio pode ter várias causas além da falta de interesse. Na maioria das vezes, o entendimento do tema é complexo, e somente posturas ativas do educador podem ajudar, como: escuta atenta a uma pergunta, respeito quando a resposta é errada, estímulo ao raciocínio para encontrar a resposta correta, elogio para respostas corretas e interrupção da simulação ou de sons a fim de permitir a interação entre discente e docente.

- Dificuldades técnicas com computadores, exceto a queda de energia ou conexão, por processadores insuficientes: Deve existir o cuidado da instituição e do docente em escolher computadores com processadores adequados antes de iniciar a sua atividade.

\section{CONCLUSÃO}

A avaliação dos especialistas indicou o software como uma ferramenta adequada para ensino da teoria e/ou prática sobre VM, e as sugestões deles contribuíram com perspectivas diferentes para melhorias do simulador.

O ensino de VM no ambiente da simulação permite ao aluno uma participação ativa no seu processo de aprendizagem. O simulador com interação cardiopulmonar desenvolvido é uma ferramenta de grande valia para alcançar o entendimento do tema, podendo despertar a curiosidade e o interesse dos discentes, respeitando a autonomia e velocidade de aprendizagem de cada um.

\section{CONTRIBUIÇÃO DOS AUTORES}

Moyses de Campos Junior é o autor do projeto de pesquisa. Suzana Guimarães Moraes contribuiu na orientação do projeto de pesquisa.

\section{CONFLITO DE INTERESSES}

Declaramos não haver conflito de interesses.

\section{FINANCIAMENTO}

Declaramos não haver financiamento.

\section{REFERÊNCIAS}

1. Lino JA, Gomes GC, Sousa NDSVC, Carvalho AK, Diniz MEB, Viana AB, et al. $A$ critical review of mechanical ventilation virtual simulators: Is it time to use them? JMIR Med Educ. 2016;2(1):e8.

2. Osaku EF. Desenvolvimento de um software didático para o apoio ao aprendizado de ventilação mecânica [dissertação]. Curitiba: Centro Federal de Educação Tecnológica do Paraná; 2005 [acesso em 11 out 2018]. Disponível em: http://repositorio.utfpr.edu.br/jspui/handle/1/110.

3. Diniz MEB. Desenvolvimento da versão 2.0 do Simulador Virtual de Ventilação Mecânica XLung [dissertação]. Fortaleza: Centro Universitário Christus; 2016 [acesso em 19 mar 2019]. Disponível em: https:// unichristus.edu.br/temis/dissertacoes/attachment/desenvolvimento-daversa\%cc\%83o-2-0-do-simulador-virtual-de-ventilac\%cc\%a7a\%cc\%83omeca\%cc\%82nica-xlung/.

4. Spadaro S, Karbing DS, Fogagnolo A, Ragazzi R, Mojoli F, Astolfi L, et al. Simulation training for residents focused on mechanical ventilation: a randomized trial using mannequin-based versus computer-based simulation. Simul Healthc. 2017;12(6):349-55.

5. Writing Group for the Alveolar Recruitment for Acute Respiratory Distress Syndrome Trial (ART) Investigators, Cavalcanti AB, Suzumura ÉA Laranjeira LN, Paisani DM, Damiani LP, et al. Effect of lung recruitment and titrated Positive End-Expiratory Pressure (PEEP) vs Low PEEP on mortality in patients with acute respiratory distress syndrome: a randomized clinical trial. JAMA. 2017;318(14):1335-45

6. Son YG, Shin J, Ryu HG. Pneumonitis and pneumonia after aspiration. J Dent Anesth Pain Med. 2017;17(1):1-12.

7. Hsu CW, Sun SF. latrogenic pneumothorax related to mechanical ventilation. World J Crit Care Med. 2014;3(1):8-14.

8. Aggarwal R, Mytton OT, Derbrew M, Hananel D, Heydenburg M, Issenberg $B$, et al. Training and simulation for patient safety. Qual Saf Health Care. 2010 Aug;19(Suppl 2):i34-43.

9. Freire P. Pedagogia da autonomia: saberes necessários à prática educativa. 57a ed. Rio de Janeiro, São Paulo: Paz e Terra; 2018.

10. Hess DR. Ventilator waveforms and the physiology of pressure support ventilation. Respir Care. 2005;50(2):166-83.

11. Hess DR. Respiratory mechanics in mechanically ventilated patients Respir Care. 2014;59(11):1773-94.

12. Barbas CSV, Ísola AM, Farias AMC, Cavalcanti AB, Gama AMC, Duarte ACM, et al. Diretrizes Brasileiras de Ventilação Mecânica - 2013. São Paulo: Associação de Medicina Intensiva Brasileira; 2013 [acesso em 2 jun 2018]. Disponível em: https://www.amib.org.br/fileadmin/user_ upload/amib/2018/junho/15/Diretrizes Brasileiras de Ventilacao Mecanica_2013_AMIB_SBPT_Arquivo_Eletronico_Oficial.pdf.

13. Munshi L, Del Sorbo L, Adhikari NKJ, Hodgson $\mathrm{CL}$, Wunsch $\mathrm{H}$, Meade $\mathrm{MO}$, et al. Prone position for acute respiratory distress syndrome. a systematic review and meta-analysis. Ann Am Thorac Soc. 2017 Oct;14(Suppl 4):S280-S288.

14. Lessa T, Pereira CAC, Soares MR, Matos R, Guimarães VP, Sanches G, et al. Reference values for pulmonary volumes by plethysmography in a Brazilian sample of white adults. J Bras Pneumol. 2019;45(3):e20180065.

15. Kopelent V, Roubik K. Effectivity of tracheal gas insufflation on artificial lung ventilation. In: Biomedical Engineering. Zurich: Acta Press; 2004, p. 417-127 [acesso em 10 jul 2020]. Disponível em: https://www. researchgate.net/publication/229056866_Effectivity_of_tracheal_gas_ insufflation_on_artificial_lung_ventilation.

16. Blanch $L$, Bernabé $F$, Lucangelo $U$. Measurement of air trapping, intrinsic positive end-expiratory pressure, and dynamic hyperinflation in mechanically ventilation patients. Respir Care. 2005;50(1):110-24.

17. Murias G, Blanch L, Lucangelo $U$. The physiology of ventilation. Respir Care. 2014;59(11):1795-807. 
18. Rubinstein I, Zamel N, DuBarry L, Hoffstein V. Airflow limitation in morbidly obese, nonsmoking men. Ann Intern Med. 1990 June 1;112(11):828-32.

19. Subirà C, de Haro C, Magrans R, Fernández R, Blanch L. Minimizing asynchronies in mechanical ventilation: current and future trends. Respir Care. 2018 Apr;63(4):464-78.

20. Höher JA. Gasto energético de pacientes em ventilação mecânica: estudo comparativo das modalidades controlada e assistida através da calorimetria indireta [tese]. Porto Alegre: Universidade Federal do Rio Grande do Sul; 2005 [acesso em 19 ago 2019]. Disponível em: https:// lume.ufrgs.br/handle/10183/5360.

21. Likert R. The method of constructing an attitude scale. New York: Wiley; 1967.

22. Moraes SG, Pereira LA. A multimedia approach for teaching human embryology: development and evaluation of a methodology. Ann Anat. 2010 Dec 20;192(6):388-95.

23. Moraes SG, Justino ML, Jansen BF, Barbosa EP, Bruno LFC, Pereira LAV. Development and validation of a strategy to assess teaching methods in undergraduate disciplines. Progress in Education. 2012;28:81-108 [acesso em 24 abr 2021]. Disponível em: http://www.novapublishers.org/catalog/ product_info.php?products_id=35562.

24. HTML Standard [acesso em 31 jul 2019]. Disponível em: https://html.spec. whatwg.org.

25. HTML Tutorial [acesso em 31 jul 2019]. Disponível em: https://www. w3schools.com/html/default.asp.

26. Associação Brasileira de Normas Técnicas [acesso em 10 fev 2019]. Disponível em: https://www.abnt.org.br

27. Associação Brasileira de Normas Técnicas. NBR ISO/IEC 9126-1. Engenharia de software - Qualidade de produto - Parte 1: Modelo de qualidade. São Paulo: ABNT; 2001 [acesso em 10 fev 2019]. Disponível em: https://www. abnt.org.br.

28. Alexandre NMC, Coluci MZO. Validade de conteúdo nos processos de construção e adaptação de instrumentos de medidas. Cien Saude Colet. 2011;16(7):3061-8.

29. Guyton AC, Hall JE. Tratado de fisiologia médica. 11a ed. Rio de Janeiro: Elsevier; 2006.
30. Oxyhemoglobin Dissociation Curve [acesso em 6 set 2019]. Disponível em: http://www.ventworld.com/resources/oxydisso/dissoc.html.

31. Amato MBP, Gonzales R, Carvalho CRR. Tomógrafo de impedância elétrica desenvolvido pela USP monitora o pulmão de pacientes em tratamento intensivo [acesso em 2 abr 2020]. Disponível em: http://www. medicinaintensiva.com.br/tomografia-impedancia-eletrica-pulmonar.htm.

32. Hutchinson D. Creating Particles in HTML5 Canvas [acesso em 17 fev 2020]. Disponível em: https://modernweb.com/creating-particles-in-html5-canvas/.

33. Invasive mechanical ventilation [acesso em 31 mar 2020]. Disponível em: https://thoracickey.com/invasive-mechanical-ventilation/.

34. Furtado S, Reis L. Inferior vena cava evaluation in fluid therapy decision making in intensive care: practical implications. Rev Bras Ter Intensiva. 2019;31(2):240-7.

35. Pinheiro BV, Pinheiro GSM, Mendes MM. Entendendo melhor a insuficiência respiratória aguda. Pulmão RJ. 2015;24(3):3-8 [acesso em 14 mar 2021]. Disponível em: http://www.sopterj.com.br/wp-content/ themes/_sopterj_redesign_2017/_revista/2015/n_03/04.pdf.

36. Barbas CSV, Bueno MAS, Amato MBP, Hoelz C, Rodrigues Junior M. Interação cardiopulmonar durante a ventilação mecânica. Rev Soc Cardiol Estado São Paulo. 1998;8(3):406-19 [acesso em 17 jun 2019]. Disponível em: https://www.researchgate.net/publication/242286832 Interacao_cardiopulmonar_durante_a_ventilacao_mecanica.

37. Leite LHR. Transporte de gases. Juiz de Fora; 2019 [acesso em $11 \mathrm{fev} 2021$ ]. Disponível em: https://www.uff.br/laura_leite/files/2019/03/Transportede-gases.pdf.

38. Reis MAS. Suporte ventilatório Covid-19. Belo Horizonte; 2020 [acesso em 21 abr 2021]. Disponível em: https://www.somiti.org.br/arquivos/site/ comunicacao/noticias/2020/covid-19/documentos/suporte-ventilatoriocovid-19-atualizado-10-abril.pdf.

39. Koenig M. FREE Sound Clips. SoundBible.com Blog [acesso em 20 jun 2020]. Disponível em: http://soundbible.com/. 